\title{
Ingesta de dióxido de cloro para la COVID-19
}

\author{
Chlorine dioxide intake for COVID-19
}

\author{
Gustavo F. Gonzales',2, \\ Cinthya Vásquez-Velásquez ${ }^{1,2,3}$
}

Gonzales GF.Vásquez-Velásquez C. Ingesta de dióxido de cloro para la COVID-19. Rev Soc Peru Med Interna. 2021;34(3): 100-106.

https://doi.org/10.36393/spmi.v34i3.609

\begin{abstract}
RESUMEN
Durante muchos años, el dióxido de cloro ha sido utilizado por su principal característica como desinfectante, abarcando diversas áreas de aplicación como la sanitaria, alimenticia, industrial, etc. La evidencia reportada hasta la fecha cita diversos estudios en modelos animales, desde revisiones sobre la toxicocinética hasta la aplicación o usos del compuesto. Su poder como bactericida, virucida y eliminador microbiológico lo convirtió en una fuente de control y potabilización de aguas.

La controversia generada actualmente es sobre el uso de este compuesto para el tratamiento y control de la COVID-19. Se ha evidenciado incongruencia con algunos resultados reportados y que su aplicación en pacientes positivos a SARS-CoV-2 o para prevenir COVID-19 puede tener efectos adversos, como un acrecentado número de intoxicaciones $y$, en otros casos, la muerte.

En la presente revisión se detalla, a partir de la evidencia científica reportada, los efectos de este compuesto sobre la salud y la interacción con el virus SARS-CoV-2.
\end{abstract}

Palabras claves: dióxido de cloro, toxicidad, COVID-19, tratamiento, metabolismo.

\section{ABSTRACT}

For many years, chlorine dioxide has been used for its main characteristic as a disinfectant, covering various areas of application such as sanitary, food, industrial, etc. The evidence reported to date cites various studies in animal models, from reviews on toxicokinetics to the application or uses of the compound. Its power as a bactericide, virucidal and microbiological eliminator made it a source of water control and purification. The controversy currently generated is about the use of this compound for the treatment and control of COVID-19. There has been evidence of inconsistency with some reported results and that its application in SARS-CoV-2 positive patients or to prevent COVID-19 may have adverse effects, such as an increased number of poisonings and, in other cases, death. In the present review it is detailed, from the scientific evidence reported, the effects of this compound on health and the interaction with SARS-Cov-2 virus.

Key words: chlorine dioxide, toxicity, COVID-19, treatment, metabolism.

I Laboratorio de Endocrinología y Reproducción, Departamento de Ciencias Biológicas y Fisiológicas, Laboratorio de Investigación y Desarrollo (LID), Facultad de Ciencias y Filosofía, Universidad Peruana Cayetano Heredia, Lima, Perú.

2 Instituto de Investigaciones de la Altura, Universidad Peruana Cayetano Heredia, Lima, Perú.

3 Unidad de Investigación, Dirección de Laboratorio de Salud Pública, Dirección Regional de Salud, Callao, Perú.

Gustavo Gonzales: https://orcid.org/0000-0003-16 I I-2894 Cinthya Vásquez: https://orcid.org/0000-0002-3326-0437

\section{INTRODUCCIÓN}

El COVID-19 es la pandemia más grave a nivel mundial desde la pandemia de gripe de 1918 y es causada por un coronavirus.

Los coronavirus (CoVs) son virus de ARN de sentido positivo no segmentados que pertenecen a la familia Coronaviridae y que contienen el genoma más grande entre los virus de ARN. ${ }^{1}$ Los coronavirus pueden generar enfermedades respiratorias leves y/o graves en humanos 
como el SARS-CoV, y el MERS-CoV. ${ }^{2}$ El SARS-CoV-2 o nuevo coronavirus recientemente descubierto en diciembre del 2019 en Wuhan-China ha generado una pandemia por su rápida propagación. ${ }^{3}$

Las interacciones entre la proteína de la envoltura del coronavirus (E) y su entorno de membrana juegan un papel clave en la estabilidad y función de la envoltura viral. ${ }^{4}$ La desintegración de la bicapa lipídica y dislocación de la proteína $\mathrm{E}$ de su entorno de membrana afecta al virus, y este mecanismo es usado por los desinfectantes para inactivarlos, tales como el alcohol ${ }^{4}$; peróxido de hidrógeno ${ }^{5}$ y sustancias cloradas. ${ }^{6,7}$

El estado de emergencia generado desde marzo 2020 por la pandemia del COVID-19 trajo consigo un fuerte impacto sanitario, social y económico en todos los países del orbe, debido a los contagios acelerados y acrecentada tasa de mortalidad. A nivel mundial, debido al poco conocimiento sobre este nuevo virus respiratorio y a la ausencia de un tratamiento eficiente, se utilizó estrategias sanitarias que incluían cuarentena, distanciamiento social, uso de mascarilla, y lavado frecuente de $\operatorname{manos}^{8,9}$. De manera paralela surgió información anecdótica para la prevención y tratamiento del COVID-19 con el uso de sustancias químicas de bajo costo y de fácil acceso. Entre ello tenemos la recomendación de tratamiento oral con dióxido de cloro, $\mathrm{ClO}_{2}$, compuesto químico usado en diversas áreas como desinfectante de agua de consumo humano, aguas residuales y conservador de alimentos; sin embargo, hasta la actualidad no se tiene un sustento fehaciente como tratamiento inocuo para la COVID-19.

La presente revisión tiene como finalidad enumerar las evidencias científicas, basadas en la literatura disponible en relación al uso del dióxido de cloro.

\section{DIÓXIDO DE CLORO COMO DESINFECTANTE}

$\mathrm{El} \mathrm{ClO}_{2}$ es un producto ampliamente utilizado en superficies, alimentos y tratamiento de aguas de consumo humano y en aguas residuales por su excelente efecto desinfectante, y como blanqueador en la industria del papel, entre otros. ${ }^{10,11}$ Así mismo, actualmente se ha demostrado su efecto como desinfectante contra el virus SARS-Cov-2. ${ }^{11-14}$

$\mathrm{El} \mathrm{ClO}_{2}$ se adsorbe a nivel de proteínas (capsómeros) de los virus, donde reacciona con el ácido ribonucleico del SARSCoV-2. Ya anteriormente se conocía sobre la eficacia del $\mathrm{ClO}_{2}$ contra el coxsackievirus B5, el bacteriófago f2, el rotavirus humano, el poliovirus1, el rotavirus del simio y el ecovirus 1. El dióxido de cloro es potente en un rango amplio de $\mathrm{pH}$, a una dosis de 1,0 mg/L. Para el SARSCoV-2 es menos efectivo que el cloro.

El dióxido de cloro puede inactivar el SARS-CoV adecuadamente después de 30 minutos de exposición y a la dosis de $40 \mathrm{mg} / \mathrm{L}$. Además, la inactivación del coronavirus murino fue exitosa poco después de la exposición al $\mathrm{ClO}_{2}$ a una dosis de $0,16 \mathrm{ppm} / \mathrm{min}$. $^{11,13} \mathrm{El} \mathrm{ClO}_{2}$ actúa desnaturalizando los aminoácidos cisteína, tirosina y triptófano, siendo mayor la reactividad con cisteína y la menor con triptófano.

Teniendo en cuenta que SARS-CoV-2 tiene 54 tirosinas, 12 triptófanos y 40 residuos de cisteína, se asume que el $\mathrm{ClO}_{2}$ en solución acuosa también actuaría sobre los residuos de proteína conteniendo estos aminoácidos y con ello podría eliminar el SARS-CoV-2. ${ }^{15}$ Las tasas de inactivación de los virus por el $\mathrm{ClO}_{2}$ aumentan a medida que aumenta el $\mathrm{pH}$ o la temperatura, pero muestran diferentes tendencias con el aumento de las concentraciones de materia orgánica disuelta (DOM). Tanto los daños en las proteínas virales como en la región no codificante 5' dentro del genoma contribuyen a la inactivación del virus tras la desinfección de $\mathrm{ClO}_{2}{ }^{12}$

\section{EXPOSICIÓN DE HUMANOS AL DIÓXIDO DE CLORO DURANTE LA DESINFECCIÓN DEL AGUA DE CONSUMO}

La desinfección significa la destrucción de microorganismos patógenos (por ejemplo: bacterias y sus esporas, virus, protozoos y sus quistes, gusanos y larvas) presentes en el agua para hacerla potable para uso doméstico. ${ }^{16}$

La cloración, debido a su eficiencia de eliminación y ser costo-efectivo, es utilizado ampliamente para la desinfección del agua. Sin embargo, el proceso de desinfección puede generar varios tipos de subproductos de desinfección (-600-700 en números) en el agua tratada, como los trihalometanos (THM), ácidos haloacéticos (HAA), etc., que son perjudiciales para los seres humanos en términos de citotoxicidad, mutagenicidad, teratogenicidad y carcinogenicidad. ${ }^{16}$ Basado en lo anterior se han establecidos varias regulaciones por organismos internacionales para proteger la salud humana. La idea es eliminar los subproductos de desinfección, así como sus precursores del agua. ${ }^{17}$

Los subproductos de desinfección se forman cuando los desinfectantes (cloro, ozono, dióxido de cloro o cloraminas) reaccionan con la materia orgánica natural, los contaminantes antropogénicos, el bromuro y el yoduro durante la producción de agua potable. ${ }^{18}$

El dióxido de cloro $\left(\mathrm{ClO}_{2}\right)$, es un desinfectante alternativo al cloro, y se aplica ampliamente en la desinfección de agua y aguas residuales. ${ }^{12}$ La manera más extensiva de exposición de dióxido de cloro en humanos es en el agua de consumo, dado su uso como desinfectante para potabilizar el agua. El dióxido de cloro no produce los problemas de sabor y olor que resultan del tratamiento con el cloro debido a que no reacciona con el fenol.

En comparación con el cloro, el $\mathrm{ClO}_{2}$ reduce en gran medida la generación de productos de desinfección halogenados tóxicos ${ }^{19}$, y el clorito y el clorato son los principales subproductos del $\mathrm{ClO}_{2}{ }^{20}$ En efecto, el dióxido de cloro es un gas muy inestable y se convierte en diferentes productos al mezclarse con el agua.

En estudios experimentales en ratas, ratones y pollos ante exposición aguda y crónica tratados con $\mathrm{ClO}_{2}, \mathrm{ClO}_{2}^{-}$y $\mathrm{ClO}_{3}^{-}$en agua potable se ha evidenciado alteraciones en parámetros hematológicos en todas las especies evaluadas. Los efectos son generalmente dosis dependiente y los cambios marcados ocurrieron en las dosis más altas (hasta $1000 \mathrm{mg} / \mathrm{l}$.). En estudios crónicos, a las ratas se les ha dado 
$\mathrm{ClO}_{2}$ a dosis de hasta $1000 \mathrm{mg} / \mathrm{l}$., y $\mathrm{NaClO}_{2}$ o $\mathrm{NaClO}_{3}$ a hasta $100 \mathrm{mg} / \mathrm{l}$., en el agua potable durante un año. Los grupos de tratamiento que recibieron $\mathrm{ClO}_{2}, \mathrm{ClO}_{2}^{-}$y $\mathrm{ClO}_{3}^{-}$ mostraron alteraciones en la morfología de los eritrocitos y fragilidad osmótica; y en las dosis más altas se produjo anemia hemolítica leve. ${ }^{21}$ Esto último se debe a que el $\mathrm{ClO}_{2}$ puede oxidar también a la hemoglobina, por lo que el ion ferroso $\left(\mathrm{Fe}^{+2}\right)$ de la hemoglobina normal pasa a ion férrico $\left(\mathrm{Fe}^{+3}\right)$ formando la metahemoglobina. Se describe en la literatura un caso de una mujer de 28 años que tomó de manera intencional nitrito de sodio y tuvo un desenlace fatal por la metahemoglobinemia. ${ }^{22}$

$\mathrm{ClO}_{2}, \mathrm{ClO}_{2}^{-} \mathrm{y} \mathrm{ClO}_{3}^{-}$alteran la incorporación de ${ }^{3} \mathrm{H}$-timidina en los núcleos de varios órganos de la rata. Estos datos sugieren la posibilidad de un aumento en la tasa de recambio de la mucosa gastrointestinal y una inhibición de síntesis de $\mathrm{ADN}$ en varios órganos. En esta última categoría, la mayor preocupación gira en torno a si la aparente disminución de la síntesis de $\mathrm{ADN}$ en los testículos se asociada con una alteración en la espermatogénesis y daño en la función reproductiva masculina. ${ }^{21}$ Falta desarrollar estudios en humanos expuestos a estos productos clorinados. Lo cierto es que en la última centuria se observa una disminución en la concentración de espermatozoides en la población masculina. ${ }^{23}$ En ratas, la administración de $10 \mathrm{mg} / \mathrm{l}$ diario de $\mathrm{ClO}_{2}$ en el agua de bebida aumentó los nioveles de cloroformo en el testículo de ratas. ${ }^{24}$

Niveles moderados de dos trihalometanos, el bromodiclorometano y el dibromoclorometano en sangre de varones se asociaron con disminución del conteo de espermatozoides y disminución de la linealidad de los espermatozoides en comparación con niveles bajos de estos compuestos, respectivamente..$^{25}$

La toxicocinética (Figura 1) de este compuesto ha sido estudiado en modelos animales usando dióxido de cloro radioactivo $\left({ }^{36} \mathrm{ClO}_{2}\right)$ se basa en una absorción a nivel gastrointestinal, epitelial o dermal, teniendo como tasa constante de absorción de 3,77/hora que es mayor que para $\mathrm{ClO}_{3}^{-}, \mathrm{ClO}_{2}^{-}$y $\mathrm{HClO}^{36} \mathrm{El}$ tiempo medio de absorción es de 0,18 horas para el dióxido de cloro. En el plasma, el dióxido de cloro tiene una vida media de 43,9 horas, el ion clorito de 35,2 horas, el ion clorato de 36,7 horas y el ácido hipocloroso de 77 horas. ${ }^{24}$

Según la Agencia de Protección del Medio Ambiente, el dióxido de cloro después de ser absorbido se distribuye ampliamente en diversos tejidos y órganos, siendo principalmente la sangre, el estómago y el intestino delgado, y menor proporción el pulmón, riñón, hígado, bazo, timo, médula ósea y testículos (estudios experimentales en modelos murino). ${ }^{26}$ Así mismo, su excreción o eliminación se da de forma eficiente en la orina y heces, y en modelo subcrónico oral de dosis pequeñas (hasta 40 ppm) no genera toxicidad en ratones. ${ }^{27}$ Estudios recientes muestran que el uso de agua desinfectada con dióxido de cloro muestra efectos mutagénicos en ensayos in vitro. ${ }^{28}$

Dado que los productos clorinados presentan toxicidad en el organismo su ingesta no es recomendada. Por ello llama la atención la propuesta de su ingesta como una forma de prevenir o de tratar al COVID-19, que ha surgido como alternativa en esta pandemia del COVID-19. La ingesta de clorito de sodio accidental al 22,4\% por un niño de 9 años le produjo efectos adversos como náuseas, vómitos, diarrea, disnea, metahemoglobinemia e insuficiencia renal que va a requerir diálisis y anemia hemolítica que va a requerir transfusión sanguínea. ${ }^{29}$ Igual ocurrió con un hombre de 65 años que accidentalmente consumió clorito de sodio. ${ }^{30}$ Un hombre en intento de suicidio ingirió $10 \mathrm{~g}$ de clorito de sodio y presentó metahemoglobinemia, anemia hemolítica e insuficiencia renal aguda. ${ }^{31}$

Los metabolitos como los cloritos y cloratos son más tóxicos que el mismo precursor $\mathrm{ClO}_{2}$ en la generación de insuficiencia renal aguda. ${ }^{32}$

\section{DIÓXIDO DE CLORO COMO ENJUAGUE BUCAL}

Se ha evaluado el dióxido de cloro para enjuague bucal y se ha demostrado que no es superior a otros enjuagues bucales. $^{33}$ Todos los enjuagues bucales tienen efectos colaterales sustanciales. ${ }^{34}$ A la fecha no hay estudios publicados sobre el uso de dióxido de cloro para enjuague bucal y/o spray nasal en los pacientes con COVID-19.35

INGESTA DE DIÓXIDO DE CLORO Y COVID-19

Toxicidad

Basado en su potente efecto desinfectante, se ha planteado que la ingesta de dióxido de cloro podría ser un método útil y de bajo costo para prevenir la propagación del COVID-19. ${ }^{36}$ Todo ello a pesar de las evidencias previas de toxicidad del dióxido de cloro y sus metabolitos a partir de su uso en el agua potable. El dióxido de cloro $\left(\mathrm{ClO}_{2}\right)$ al ser ingerido y reaccionar en el agua forma iones de clorito $\left(\mathrm{ClO}_{2}^{-}\right)$que son sustancias aún más reactivas que el mismo dióxido de cloro. ${ }^{37}$

En Estados Unidos salió un producto, ahora prohibido, conocido como solución mineral milagrosa en base de clorito de sodio al $28 \%$ que se comercializa para el tratamiento del COVID-19. ${ }^{38}$ Este producto aparece hace más de una década clamando efectos importantes en diferentes enfermedades. Los reportes sobre efectos adversos ya han sido descritos en la literatura; así en 2014 se reporta un caso de enfermedad de Kikuchi-Fujimoto en una mujer de 41 años por consumir dichos productos. ${ }^{39}$ Según su creador Jim Humble, el producto debe mezclar tres gotas de la solución de clorito de sodio con una solución ácida como jugo de limón para formar dióxido de cloro.

Se ha publicado un análisis de los casos del Sistema Nacional de Datos sobre Venenos (NPDS) de la Asociación Americana de Centros de Control de Envenenamiento (AAPCC de las siglas en inglés) de la exposición asociada a dióxido de cloro (que implica la solución mineral milagrosa) de 55 centros de venenos de los E.E.U.U. entre el 1 de enero de 2000-31 de marzo de 2020. Se presentan narrativas para 53 exposiciones con dióxido de cloro. Se reportó un promedio de cinco exposiciones anuales (rango 3-9) desde 2011. En $85 \%$ de casos, los efectos adversos ocurrieron inmediatamente o dentro de las $24 \mathrm{~h}$ de exposición; $25 \%$ fueron hospitalizados. ${ }^{40}$ 
En países de la región como Argentina y en el Ecuador se han notificado un incremento de casos por intoxicación, debido al uso de desinfectantes, como el dióxido de cloro, para el tratamiento de COVID-19. ${ }^{41}$ Igualmente, una reciente publicación en una revista indizada y revisada por pares demuestra en un varón de 55 años que la ingesta de dióxido de cloro para prevenir la COVID-19 resultó en insuficiencia renal aguda y coagulación intravascular diseminada, que revirtieron luego de la hemodiálisis. ${ }^{42} \mathrm{Su}$ cuadro lo inició luego de cnco días del uso del dióxido de cloro con náuseas, vómitos, y estado mental alterado.

Diferentes instituciones a nivel mundial ${ }^{43}$, así como la Food and Drug Administration ${ }^{44,45}$ (FDA) de los Estados Unidos y la Organización Panamericana de la Salud ${ }^{46}$ han alertado sobre el peligro del uso por vía oral del dióxido de cloro o similares para la prevención o para el tratamiento del COVID-19. En el Perú, el Instituto Nacional de Salud (INS) y EsSalud han emitido sendos reportes recomendando no usar el dióxido de cloro o similares para el tratamiento de pacientes con diagnóstico de COVID-19. ${ }^{47,48}$

La FDA ha reportado de personas con eventos adversos graves luego de consumir productos de dióxido de cloro. Estos efectos adversos incluyen a la insuficiencia respiratoria causada por una condición grave donde la cantidad de oxígeno transportado en la sangre se reduce notablemente (metahemoglobinemia); a cambios en la actividad eléctrica del corazón (prolongación de QT), que pueden conducir a anormalidad en los ritmos cardíacos que pueden llegar a ser fatales; una reducción en la presión arterial potencialmente mortal que causada por la deshidratación; insuficiencia hepática; recuentos bajos de glóbulos rojos debido a hemólisis (anemia hemolítica), que requirió una transfusión de sangre; vómitos severos; y diarrea severa.

\section{Eficacia contra COVID-19}

Al 7 de julio de 2021, no hay ninguna publicación científica en revistas indizadas y evaluadas por pares que demuestren que la ingesta por vía oral del dióxido de cloro o derivados es eficaz como tratamiento o como profilaxis contra el COVID-19. ${ }^{37-49}$ En nuestra búsqueda bibliográfica hemos encontrado una publicación en una revista no indizada basado en un ensayo clínico el cual ha sido registrado en Clinical trials.gov con número NCT04343742 (Determination of the Effectiveness of oral chlorine dioxide in the treatment of COVID 19) ${ }^{50}$ Este estudio tiene numerosas falencias y baja calidad científica.

Según el registro en el clinical trial.Gov, el estudio fue aprobado para ser realizado en Bogotá (Colombia) y Madrid (España), lo que no se ha cumplido en el estudio publicado pues incluyen pacientes de Bolivia, Perú, Ecuador y México, lo cual de por sí revela una falta a la ética. Los pacientes del grupo experimental que recibieron dióxido de cloro son de Bolivia (catorce pacientes), Perú (dos pacientes) y Ecuador (cuatro pacientes). El grupo control fue de Ecuador (ocho pacientes), Bolivia (siete pacientes), México (tres pacientes) y Perú (dos pacientes). No se entiende como México contribuye con dos casos del grupo control y ninguno para el grupo experimental. Existen diferencias en el manejo sanitario entre países; igualmente las condiciones hospitalarias difieren entre países y aún entre regiones de un mismo país. ${ }^{51,52}$ Esto demuestra un inadecuado apareamiento pues las condiciones de un ensayo caso-control requiere que las condiciones en ambos grupos sean exactamente igual excepto la intervención que es la que se va a evaluar. Este estudio incluye 20 sujetos con COVID-19 en el grupo control y 20 sujetos que reciben dióxido de cloro (3000 ppm) en agua como grupo experimental. El grupo control tienen valores significativamente más altos de proteína $\mathrm{C}$ reactiva ( $\mathrm{PCR}$ ) al inicio del estudio que el grupo tratado con dióxido de cloro $(p=0.0398)$ por lo que no son comparables desde el inicio. Se ha demostrado que en pacientes hospitalizados por COVID-19 aquellos con niveles elevados de proteína C reactiva tienen un peor pronóstico que aquellos con valores menores. ${ }^{53} \mathrm{Si}$ los grupos de estudio con y sin intervención no son comparables, en este caso por tener diferentes valores de marcadores inflamatorios al inicio del estudio no permiten tener conclusiones adecuadas de los resultados del estudio. La publicación de Insignares-Carrione y col..$^{50}$ no menciona cuales fueron los criterios utilizados para asignar a un sujeto como control y a otro para el tratamiento con dióxido de cloro. El estudio concluye que el dióxido de cloro es efectivo en el tratamiento de COVID-19 haciendo RTPCR negativo en $100 \%$ de casos al día 7 , y modificando los síntomas de la enfermedad significativamente y reduciendo los parámetros de laboratorio a normalidad dentro de los 14 y 21 días. Es de notar que el grupo control recibió tratamiento antiinflamatorio (ibuprofeno a dosis entre 200 y $400 \mathrm{mg}$ cada 8 horas), antibióticos (azitromicina $500 \mathrm{mg}$ diarios durante cinco días), antihistamínicos (hidroxizina $5 \mathrm{mg}$ cada 12 horas), corticosteroides (generalmente 40 $\mathrm{mg}$ de metilprednisolona cada 12 horas durante tres días y luego $20 \mathrm{mg}$ cada 12 horas durante tres días) y medidas de apoyo. El grupo experimental no recibió este tratamiento ${ }^{50}$. Se conoce en la actualidad que el uso temprano y de corto plazo del corticosteroide prolongan la duración de detección de carga viral en la garganta y prolonga el síndrome respiratorio agudo grave por coronavirus 2 en pacientes con neumonía por COVID-19. ${ }^{54}$ Por lo tanto, se podría sugerir que el mayor tiempo de carga viral y mayor sintomatología y alteración de pruebas de laboratorio se deba al uso temprano de corticoides en el grupo control. Por lo expuesto, los resultados de este estudio deben ser tomados con cautela.

Los ensayos clínicos deben seguir pautas metodológicas para asegurar su validez interna y externa. Para ello deben cumplir con ciertos requerimientos establecido como la Declaración de CONSORT (Consolidated Standards of Reproting Trials). ${ }^{55}$ Para el caso de la pandemia de COVID-19 se han establecido nuevas guías que adecuan el listado de CONSORT. ${ }^{56}$ La Declaración de CONSORT proporciona un conjunto estándar mínimo de elementos que deben ser reportados en los ensayos clínicos publicados. ${ }^{57}$ Para buscar una analogía al uso de un desinfectante como 


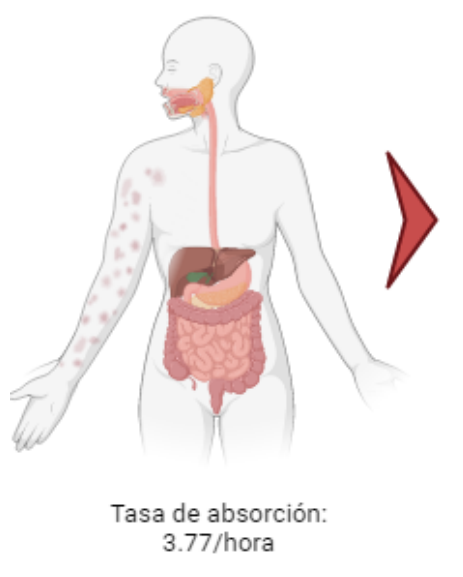

Absorción

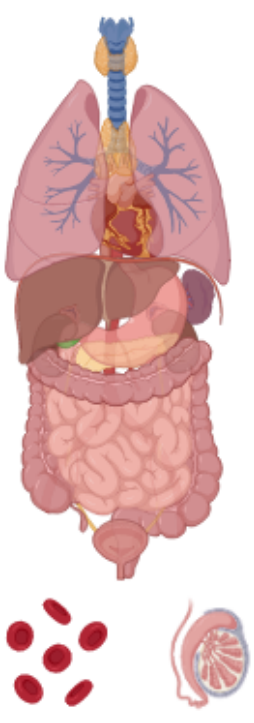

Distribución

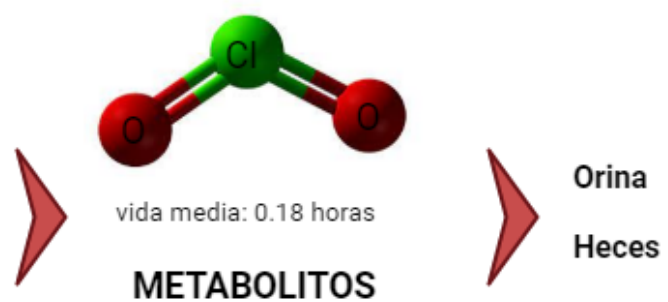

Clorito

Cloruro

Clorato

Figura I. Toxicocinética del dióxido de cloro $\left(\mathrm{ClO}_{2}\right)$. Fuente: 24 .

dióxido de cloro, útil para destruir el SARS-CoV-2 en las superficies pero tóxico cuando se ingiere, podemos decir que el peróxido de hidrógeno es eficaz para el SARS-CoV-2 como desinfectante ${ }^{5}$ pero a nadie se le ocurriría tomar agua oxigenada por su toxicidad. ${ }^{58}$

En conclusión, no hay evidencia científica que avale su ingesta oral para prevención ni tratamiento de ninguna enfermedad incluida el COVID-19. A partir de la evidencia notificada se exhorta a la población peruana no ingerir dióxido de cloro y que la comercialización de este desinfectante para ingesta oral o parenteral con fines preventivos o terapéuticos para COVID-19 deben ser denunciada a las autoridades competentes.

\section{REFERENCIAS BIBLIOGRÁFICAS}

I. Najafi Fard S, Petrone L, Petruccioli E,Alonzi T, Matusali G, Colavita $F$, et al. In vitro models for studying entry, tissue tropism, and therapeutic approaches of highly pathogenic coronaviruses. Biomed Res Int. 2021 Jun 21;2021:8856018. doi: 10.1155/2021/88560I8. PMID: 34239932; PMCID: PMC822 I88।

2. Cui J, Li F, Shi ZL. Origin and evolution of pathogenic coronaviruses. Nat Rev Microbiol. 2019 Mar;I7(3):I8I-192. doi: I0.1038/s4I5790I8-0II8-9. PMID: 3053 I947; PMCID: PMC7097006.

3. Li H, Zhou Y, Zhang M,Wang H, Zhao Q, Liu J. Updated Approaches against SARS-CoV-2. Antimicrob Agents Chemother. 2020 May 2I;64(6): e00483-20. doi: I0.I I28/AAC.00483-20. PMID: 32205349; PMCID: PMC72695I 2.

4. Das S, Meinel MK, Wu Z, Müller-Plathe F. The role of the envelope protein in the stability of a coronavirus model membrane against an ethanolic disinfectant. J Chem Phys. 202I Jun 28;I54(24):245I0I. doi: I0.1063/5.005533I. PMID: 3424I335.

5. Mohanan PV, Sangeetha V, Sabareeswaran A, Muraleedharan V, Jithin $\mathrm{K}$,Vandana U,Varsha SB. Safety of $0.5 \%$ hydrogen peroxide mist used in the disinfection gateway for COVID-19. Environ Sci Pollut Res Int. 202 I Jul 7:I-II. doi: I0.1007/s II356-02I-I5I64-y. Epub ahead of print. PMID: 34232429; PMCID: PMC826035I.

6. Farahmandfar R, Asnaashari M, Hesami B. Monitoring of new coronavirus (SARS-CoV-2): Origin, transmission, and food preservation methods. J Food Process Preserv. 202I Jun 12:el5564. doi: I0.IIII/jfpp.I5564. Epub ahead of print. PMID: 34219846; PMCID: PMC82370I 3.

7. Takeda Y, Matsuda S, Jamsransuren D, Ogawa H. Comparison of the SARS-CoV-2-inactivating activities of the differently manufactured hypochlorous acid water products with various $\mathrm{pH}$.J Water Health. 202I Jun;19(3):448-456. doi: I0.2166/wh.2021.260. PMID: 34I52297.

8. Farman M,Aslam M,Akgül A,Ahmad A. Modeling of fractional-order COVID-I9 epidemic model with quarantine and social distancing. Math Methods Appl Sci. 202 I Jul 30;44(I I):9334-9350. doi: I0.1002/ mma.7360. Epub 202I Mar 29. PMID: 34230734; PMCID: PMC825I478.

9. Al-Shaikh A, Mahafzah BA, Alshraideh M. Hybrid harmony search algorithm for social network contact tracing of COVID-19. Soft comput. 202 I Jun 28:I-23. doi: 10.1007/s00500-02I-05948-2. Epub ahead of print. PMID: 3422030 I; PMCID: PMC8237257.

10. Han S, Jo JY, Park SR, Choi C, Ha SD. Impact of chlorine dioxide and electron-beam irradiation for the reduction of murine norovirus in low-salted "jogaejeotgal", a traditional Korean salted and fermented clam. Int J Food Microbiol. 202I Mar 16;342:109073. doi: 10.1016/j.ijfoodmicro.2021.109073.

II. Shimabukuro PMS, Duarte ML, Imoto AM, Atallah ÁN, Franco ESB, Peccin MS, y col. Environmental cleaning to prevent COVID-19 infection. A rapid systematic review. Sao Paulo Med J. 2020 NovDec;|38(6):505-5|4. doi: I0.|590/|5|6-3|80.2020.04|7.09092020. PMID: 33206913.

12. Ge Y, Zhang X, Shu L, Yang $X$. Kinetics and Mechanisms of Virus Inactivation by Chlorine Dioxide in Water Treatment:A Review. Bull Environ Contam Toxicol. 202I Apr;106(4):560-567. doi: 10.1007/ s00I28-02I-03।37-3.

13. Singh S, Kumar V, Kapoor D, Dhanjal DS, Bhatia D, Jan S, et al. Detection and disinfection of COVID-19 virus in wastewater. Environ Chem Lett. 202I Feb 22:I-I7. doi: 10.1007/sI03II-02I0I202-I.

14. Hassenberg K, Praeger U, Herppich WB. Effect of chlorine dioxide treatment on human pathogens on iceberg lettuce. Foods. 202I Mar 10;10(3):574. doi: 10.3390/foodsI0030574. PMID: 3380I806; PMCID: PMC800I664

15. Kály-Kullai K, Wittmann M, Noszticzius Z, Rosivall L. Can chlorine dioxide prevent the spreading of coronavirus or other viral infections? Medical hypotheses. Physiol Int. 2020 Mar I;I07(I):I-II. 
doi: 10.1556/2060.2020.000|5.

16. Srivastav AL, Patel N, Chaudhary VK. Disinfection by-products in drinking water: Occurrence, toxicity and abatement. Environ Pollut. 2020 Dec;267:II5474. doi: 10.1016/j.envpol.2020.II5474. Epub 2020 Aug 23. PMID: 32889516.

17. Kali S, Khan M, Ghaffar MS, Rasheed S, Waseem A, lqbal MM, et al. Occurrence, influencing factors, toxicity, regulations, and abatement approaches for disinfection by-products in chlorinated drinking water:A comprehensive review. Environ Pollut.202 I Jul I 5;28 I: I I 6950. doi: I0.10I6/j.envpol.202I.II6950. Epub 202I Mar I7. PMID: 33819670

18. Richardson SD, Plewa MJ, Wagner ED, Schoeny R, Demarini DM. Occurrence, genotoxicity, and carcinogenicity of regulated and emerging disinfection by-products in drinking water: a review and roadmap for research. Mutat Res. 2007 Nov-Dec;636(I-3): I 78-242. doi: 10.1016/j.mrrev.2007.09.00I. Epub 2007 Sep 12. PMID: I7980649.

19. Zhong Y, Gan W, Du Y, Huang H, Wu Q, Xiang Y, Shang C, Yang X Disinfection byproducts and their toxicity in wastewater effluents treated by the mixing oxidant of $\mathrm{ClO}_{2} / \mathrm{Cl}_{2}$. Water Res. $2019 \mathrm{Oct}$ I;|62:47|-48I. doi: I0.10I6/j.watres.2019.07.0I2. Epub 2019 Jul 6. PMID: 3 I 302364.

20. Sorlini S, Gialdini F, Biasibetti M, Collivignarelli C. Influence of drinking water treatments on chlorine dioxide consumption and chlorite/chlorate formation. Water Res. 20/4;54:44-52. doi: 10.1016/j.watres.2014.01.038.

21. Couri D,Abdel-Rahman MS, Bull RJ.Toxicological effects of chlorine dioxide, chlorite and chlorate. Environ Health Perspect. 1982 Dec;46:13-7. doi: 10.1289/ehp.8246|3. PMID: 6759I07; PMCID: PMCI569035.

22. Huntington GR, Pennington JM. Fatal methaemoglobinaemia due to intentional sodium nitrite poisoning. Acute Med. 2021;20(2): I48I50. PMID: 34 I 90743.

23. Levine H, Jørgensen N, Martino-Andrade A, Mendiola J, WekslerDerri D, Mindlis I, Pinotti R, Swan SH. Temporal trends in sperm count: a systematic review and meta-regression analysis. Hum Reprod Update. 2017 Nov I;23(6):646-659. doi: 10.1093/humupd/dmx022. PMID: 2898I654; PMCID: PMC6455044.

24. Abdel-Rahman MS, Couri D, Bull RJ. Metabolism and pharmacokinetics of alternate drinking water disinfectants. Environ Health Perspect. I982 Dec;46: 19-23. doi: I0.1289/ehp.8246I9. PMID: 7I5I76I; PMCID: PMCI569026.

25. Zeng Q, Li M, Xie SH, Gu LJ,Yue J, Cao WC, Zheng D, Liu AL, Li YF, Lu WQ. Baseline blood trihalomethanes, semen parameters and serum total testosterone: a cross-sectional study in China. Environ Int. 2013 Apr;54:134-40. doi: 10.1016/j.envint.2013.01.016. Epub 2013 Feb 26. PMID: 23454109.

26. U.S. Environmental Protection Agency. Toxicological review of chlorine dioxide and chlorite. Support of summary information on the Integrated Risk Information System (IRIS). Washington, DC. EPA/636/R-00/007; 2000

27. Ma JW, Huang BS, Hsu CW, Peng CW, Cheng ML, Kao JY, y col. Efficacy and Safety Evaluation of a Chlorine Dioxide Solution Int J Environ Res Public Health. 2017 Mar 22;14(3):329. doi: 10.3390/ ijerph 14030329.

28. Feretti D,Acito M, Dettori M, Ceretti E, Fatigoni C, Posadino S, et al. Genotoxicity of source, treated and distributed water from four drinking water treatment plants supplied by surface water in Sardinia, Italy. Environ Res. 2020 Jun; 185:109385. doi: 10.1016/j. envres.2020.109385.

29. Zhen J, Hakmeh W. Siblings with pediatric sodium chlorite toxicity causing methemoglobinemia, renal failure and hemolytic anemia.Am J Emerg Med. 2021 Apr;42:262.e3-262.e4. doi: 10.1016/j. ajem.2020.09.003. Epub 2020 Sep 8. PMID: 32948394.

30. Romanovsky A, Djogovic D, Chin D. A case of sodium chlorite toxicity managed with concurrent renal replacement therapy and red cell exchange. J Med Toxicol. 2013 Mar;9(I):67-70. doi: I0.1007/ s।3I8I-0I2-0256-9. PMID: 22996 I35; PMCID: PMC3576492.

3I. Lin JL, Lim PS.Acute sodium chlorite poisoning associated with renal failure. Ren Fail. 1993; I5(5):645-8. doi: I0.3 109/088602293090694I 7
PMID: 82907I 2

32. Bathina G, Yadla M, Burri S, Enganti R, Prasad Ch R, Deshpande $P$, et al. An unusual case of reversible acute kidney injury due to chlorine dioxide poisoning. Ren Fail. 2013 Sep;35(8): I I76-8. doi: |0.3 I09/0886022X.20 |3.8|97| I.Epub 20|3 Aug I.PMID:2390229|

33. Mohammad AR, Giannini PJ, Preshaw PM, Alliger H. Clinical and microbiological efficacy of chlorine dioxide in the management of chronic atrophic candidiasis: an open study. Int Dent J. 2004 Jun;54(3):I54-8. doi: I0.IIII/j.|875-595x.2004.tb00272.x. PMID: 15218896.

34. Kerémi B, Márta K, Farkas K, Czumbel LM, Tóth B, Szakács Z, et al Effects of chlorine dioxide on oral hygiene - A systematic review and meta-analysis. Curr Pharm Des. 2020;26(25):3015-3025. doi: 10.217 4/I38I6I28266662005 I5I34450. PMID: 324I 0557.

35. Burton MJ, Clarkson JE, Goulao B, Glenny AM, McBain AJ, Schilder AG, y col. Use of antimicrobial mouthwashes (gargling) and nasal sprays by healthcare workers to protect them when treating patients with suspected or confirmed COVID-19 infection. Cochrane Database Syst Rev. 2020 Sep 16;9:CD0 I 3626. doi: I0. I002/I465 I858. CD0I3626.pub2. PMID: 32936949.

36. Karnik-Henry MS. Acidified sodium chlorite solution: A potential prophylaxis to mitigate impact of multiple exposures to COVID-19 in frontline health-care providers. Hosp Pract (1995). 2020 Oct;48(4): I65-I68. doi: I0.1080/2। 548331.2020.1778908.

37. Yang B, Fang H, Chen B, Yang S, Ye Z, Yu J. Effects of reductive inorganics and NOM on the formation of chlorite in the chlorine dioxide disinfection of drinking water. J Environ Sci (China). 202I Jun; 104:225-232. doi: 10.1016/j.jes.2020.1 I.033. Epub 2020 Dec 22. PMID: 33985725.

38. NewsCAP: The FDA warns consumers not to drink Miracle Mineral Solution and other sodium chlorite products. Am J Nurs. 2019 Dec;II9(I2):I4. doi: I0.I097/0I.NAJ.00006I5732.I65I6.93. PMID: 3 I 764037

39. Loh JM, Shafi H. Kikuchi-Fujimoto disease presenting after consumption of 'Miracle Mineral Solution' (sodium chlorite). BM Case Rep. 2014 Nov 24;20|4:bcr20|4205832. doi: 10.II36/bcr20I4-205832. PMID: 2542233I; PMCID: PMC424435I.

40. Lardieri A, Cheng C, Jones SC, McCulley L. Harmful effects of chlorine dioxide exposure. Clin Toxicol (Phila). 202I May;59(5):448449. doi: 10.1080/15563650.2020.1818767.

4I. ServiciolntegradodeSeguridadECU9II. 2020. URL disponible en: https://www.ecu9| I.gob.ec/al-9-I-I-se-reportaron-I0I-casos-porintoxicacion-con-productos-de-limpieza-a-escala-nacional/

42. Medina-Avitia E, Tella-Vega P, García-Estrada C. Acute kidney injury secondary to chlorine dioxide use for COVID-19 prevention. Hemodial Int. 202 I Jun 3: I0. I I I I/hdi.I294I. doi: I0.I I I I/hdi. I294 I. Epub ahead of print. PMID: 34085396; PMCID: PMC82398I5.

43. Modujovich-Buschiazzo P, Marin G, Dorati C. Alerta sobre dióxido de cloro. Centro Universitario de Farmacología, La Plata, Argentina. 202I: I-7.

44. FDA. Coronavirus (COVID-19) update: FDA warns seller marketing dangerous chlorine dioxide products that claim to treat or prevent COVID-19. https://www.fda.gov/news-events/press-announcements /coronavirus-covid-I9-update-fda-warns-seller-marketingdangerous-chlorine-dioxide-products-claim. 2020

45. FDA. Warning letter. https://www.fda.gov/inspections-complianceenforcement-and-criminal-investigations/warning-letters/crownwellness-inc-6I0844-0429202I.2021

46. OPS. 2020. URL disponible en:https://iris.paho.org/handle/ I 0665.2/52484\#: :text=La\%200rganizaci\%C3\%B3n\%20 Panamericana\%20de\%20la,o\%20inhalaci\%C3\%B3n\%20de\%20 estos\%20productos.

47. EsSalud. Uso de dióxido de cloro para el tratamiento de pacientes con diagnóstico de COVID-19. Instituto de Evaluación de Tecnologías en Salud e Investigación. 2020.

48. INS. Eficacia y seguridad del dióxido de cloro para el tratamiento de COVID-19. Unidad de Análisis y Generación de Evidencias en Salud Pública. 2020

49. Burela A, Hernández-Vásquez A, Comandé D, Peralta V, Fiestas F. Chlorine dioxide and chlorine derivatives for the prevention or 
treatment of COVID- 19: a systematic review. Rev Peru Med Exp Salud Publica. 2020 Oct-Dec;37(4):605-6I 0. Spanish, English. doi: I0. I 7843/ rpmesp.2020.374.6330.

50. Insignares-Carrione E, Bolano Gomez B, Andrade $Y$, Callisperis $P$, Suxo AM, Ajata San Martin AB, y col. Determination of the Effectiveness of Chlorine Dioxide in the Treatment of COVID-19.J Mol Genet Med. 202I; I5: S2 doi: I0.3742I/ jmgm.202I.I5.48I

5I. Hao R, Zhang Y, Cao Z, Li J, Xu Q, Ye L, Guo X, Zheng T, Song H. Control strategies and their effects on the COVID-I9 pandemic in 2020 in representative countries. J Biosaf Biosecur. 2021 Jun 25. doi: 10.1016/j.jobb.2021.06.003. Epub ahead of print. PMID: 34I89427; PMCID: PMC8226067.

52. Mattiuzzi C, Lippi G, Henry BM. Healthcare indicators associated with COVID-19 death rates in the European Union. Public Health. 202I Apr; 193:41-42. doi: 10.1016/j.puhe.2021.01.027. Epub 202I Feb II.PMID: 33725494; PMCID: PMC7877209.

53. Balushi AA, AIShekaili J, Kindi MA, Ansari Z, Al-Khabori M, Khamis $\mathrm{F}$, et al. Immunological predictors of disease severity in patients with COVID-19. Int J Infect Dis. 202I Jun 30:SI20I-97I2(2I)00546-4. doi: I0.10I6/j.ijid.2021.06.056. Epub ahead of print. PMID: 342I6735; PMCID: PMC82453I0.

54. Tang X, Feng YM, Ni JX, Zhang JY, Liu LM, Hu K, et al. Early use of corticosteroid may prolong SARS-CoV-2 shedding in nonintensive care unit patients with COVID-19 pneumonia: A multicenter, single-blind, randomized control trial. Respiration. 202I;I00(2):II6-I26. doi: I0.II59/0005I2063.

55. Altman DG, Moher D, Schulz KF. Improving the reporting of randomised trials: the CONSORT Statement and beyond. Stat Med. 2012 Nov 10;3 I (25):2985-97. doi: I0.1002/sim.5402. Epub 20I 2 Aug I8. PMID: 22903776.
56. Orkin AM, Gill PJ, Ghersi D, Campbell L, Sugarman J, Emsley R, et al. Guidelines for Reporting Trial Protocols and Completed Trials Modified Due to the COVID-19 Pandemic and Other Extenuating Circumstances:The CONSERVE 202I Statement.JAMA. 202I Jun 21. doi: I0.100I/jama.202I.994I. Epub ahead of print. PMID: 34 I 52382

57. Shamseer L, Hopewell S, Altman DG, Moher D, Schulz KF. Update on the endorsement of CONSORT by high impact factor journals: a survey of journal "Instructions to Authors" in 20I4. Trials. 2016 Jun 24;I7(I):30I. doi: I0.II86/s|3063-0I6-|408-z. PMID: 27343072; PMCID: PMC4921029.

58. Lee JS, Cha YS. Application of a new hyperbaric oxygen therapy protocol in patients with arterial and venous gas embolism due to hydrogen peroxide poisoning. Undersea Hyperb Med. 2021 Second-Quarter;48(2):I87-I93. PMID: 339754I0.

59. Quibar, Juan. Cuando la desinformación mata: el dióxido de cloro ya habría provocado 2 muertes y 2 intoxicaciones en el país. 16 agosto del 2020. URL disponible en: https://chequeado.com/el-explicador/ cuando-la-desinformacion-mata-el-dioxido-de-cloro-ya-habriaprovocado-dos-muertes-y-dos-intoxicaciones-en-el-pais/

\section{CORRESPONDENCIA:}

Gustavo Gonzales

gustavo.gonzales@upch.pe

Fecha de recepción: |3-07-202|.

Fecha de aceptación: 20-08-202I.

Conflicto de interés: ninguno, según los autores.

Financiamiento: por los autores. 